Check for updates

Cite this: RSC Adv., 2017, 7, 50578

\title{
Elastic FRET sensors for contactless pressure measurement $\uparrow$
}

\author{
Frank Morgner, ${ }^{a c}$ Mark Bennemann, $\dagger^{a}$ Piotr J. Cywiński, ${ }^{a}$ Matthias Kollosche, ${ }^{b}$ \\ Krzysztof Górski, ${ }^{d}$ Marek Pietraszkiewicz, (D) André Geßner (ID *a \\ and Hans-Gerd Löhmannsröben ${ }^{c}$
}

Contactless pressure monitoring based on Förster resonance energy transfer between donor/acceptor pairs immobilized within elastomers is demonstrated. The donor/acceptor energy transfer is employed by dispersing terbium(III) tris[(2-hydroxybenzoyl)-2-aminoethyl] amine complex (LLC, donor) and CdSe/ ZnS quantum dots (QD655, acceptor) in styrene-ethylene/buthylene-styrene (SEBS) and poly(dimethylsiloxane) (PDMS). The continuous monitoring of QD luminescence showed a reversible intensity change as the pressure signal is alternated between two stable states indicating a pressure sensitivity of $6350 \mathrm{cps} \mathrm{kPa}{ }^{-1}$. Time-resolved measurements show the pressure impact on the FRET signal due to an increase of decay time (270 $\mu$ s up to $420 \mu \mathrm{s})$ for the donor signal and parallel drop of decay time $(170 \mu \mathrm{s}$ to $155 \mu \mathrm{s})$ for the acceptor signal as the net pressure applied. The LLC/QD655 sensors enable a contactless readout as well as space resolved monitoring to enable miniaturization towards smaller integrated stretchable opto-electronics. Elastic FRET sensors can potentially lead to developing profitable analysis systems capable to outdo conventional wired electronic systems (inductive, capacitive, ultrasonic and photoelectric sensors) especially for point-of-care diagnostics, biological monitoring required for wearable electronics.

Received 7th June 2017

Accepted 9th October 2017

DOI: $10.1039 / c 7 r a 06379 b$

rsc.li/rsc-advances structures for real-time damage diagnosis and localization. ${ }^{12}$ However, any commercial success of flexible technologies depends not only on factors such as reliability and reproducibility, but also on the scope for miniaturization and integration of components for $2 \mathrm{D}^{13}$ and $3 \mathrm{D}^{14,15}$ flexible architectures. A quite common approach is the use of existing materials as elastomeric substrate for rigid electronic components ${ }^{16}$ with dramatic increase in engineering effort and multi-step fabrication, partially to ensure reliable interconnects for power supply and signal readout. However, a complementary strategy to reduce complexity is to enhance the intrinsic materials responses by nanoparticles ${ }^{17}$ or on the molecular level ${ }^{18}$ but also to inscribe components directly into elastomer films. ${ }^{19,20}$

Hence, dispersed nanosensors, ${ }^{21}$ nanoparticles acting as signal generator and detector within an elastomer may combines both strategies. Non-contact optical methods could allow for displacement, force, chemical as well as biological monitoring and may pave the way towards more compact multiplex sensors with tailored sensitivity and efficiency. Among various sensing mechanisms, Förster resonance energy transfer (FRET) ${ }^{22-24}$ a radiationless energy transfer between two molecules (donor and acceptor) being in vicinity of several nanometres, have gained tremendous popularity in biology, biochemistry, medicine, and life sciences. ${ }^{25-28}$ The FRET efficiency $\eta_{\text {FRET }}$ strongly depends on the donor/acceptor distance $d\left(\eta_{\text {FRET }} \sim d^{-6}\right)$ which enables bioanalytical measurements of 
molecular systems in the range of $1 \mathrm{~nm}$ to $10 \mathrm{~nm}$ i.e. below light diffraction limits. ${ }^{29,30}$ In addition to simultaneous measurement of multiple processes ${ }^{31}$ this effect allows for full optic excitation and readout and could enable novel elastic sensors with contactless readout.

In this work, a FRET pair combination of luminescent lanthanide complex (LLCs) (donor) and semiconductor quantum dots (QDs) (acceptor) dispersed in an elastomeric host is exposed to an alternating pressure, which causes the FRET sensor to deform biaxially in the apex region and thus leads to an increase of the average donor/acceptor distance and decrease of the FRET efficiency, respectively.

\section{Experimental section}

\section{Synthesis of the terbium complex}

The luminescent terbium complex (LLC) (donor) was synthesized according to published protocols. ${ }^{32,33}$ Details relevant for the present investigation are discussed briefly. The ligand was synthesized as presented in Scheme 1: tris(2-ethylamino) amine (TREN, $5 \mathrm{~mL}$ ) was dissolved in an excess of methyl salicylate (30 $\mathrm{mL}$ ), and heated under reflux for $10 \mathrm{~h}$. After cooling, the mixture was diluted with $100 \mathrm{~mL}$ ethyl ether and the product precipitated off as a white solid. The product was filtered, washed with ethyl ether $(2 \times 50 \mathrm{~mL})$, dried in air and purified twice by recrystallization from methanol. This treatment yields up to $10.6 \mathrm{~g}(63 \%)$ of the ligand. MS ESI (MeOH): $507.4(\mathrm{M}+1), 529(\mathrm{M}$ $\left.+\mathrm{Na}^{+}\right)$. The $\mathrm{Tb}(\mathrm{III})$ complex was synthesized as sketched in Scheme 2 . Here, the ligand (1016 mg, $2 \mathrm{mmol}$ ) was dissolved in $50 \mathrm{~mL}$ methanol and the terbium trifluoromethanesulfonate (1212 $\mathrm{mg}, 2 \mathrm{mmol}$ ) was added under rigorous stirring. The triethylamine was added rapidly $(0.84 \mathrm{~mL}, 6 \mathrm{mmol})$ and a white precipitate started to form. The mixture was stirred at reflux for $5 \mathrm{~h}$, cooled, filtered and washed with methanol $(2 \times 10 \mathrm{~mL})$. The product was dried in vacuum desiccator over $\mathrm{P}_{2} \mathrm{O}_{5}$ and yielded $1115 \mathrm{mg}$ of the LLC. All chemicals used were purchased either from Sigma-Aldrich (Poznan, Poland) or Wistol Solvent (Oswiecim, Poland).

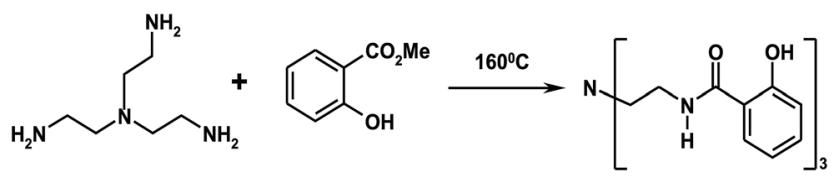

Scheme 1 The synthesis of the ligand used to prepare terbium complex.

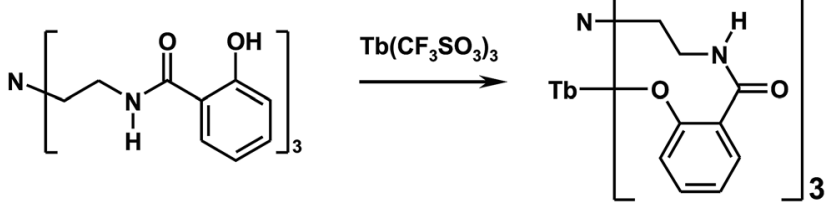

Scheme 2 The synthesis of the Tb(III) complex used to prepare FRETbased pressure sensor.

\section{Sensor preparation}

FRET is based on dipole-dipole coupling, which requires spectral overlap between donor fluorescence emission and acceptor absorbance (see Fig. 1). ${ }^{34,35}$ To match the LLC emission, quantum dots QD655 $1 \mu \mathrm{M}$ solution (Qdot ${ }^{\circledR} 655$ ITK $^{\mathrm{TM}}$, Life Technologies (Carlsbad, USA)) were used. Styrene-ethylene/buthylene-styrene copolymer (SEBS 500.120 M, HEXPOL TPE AB (Sweden)) and poly(dimethylsiloxane) (PDMS, Sylgard 184, Dow Corning (Auburn, USA)) are selected for dispersing QD655 and LLC as well as for sensor encapsulation. All elastomers are assumed to be incompressible, $L_{1} L_{2} L_{3}=1$, where $L_{1}, L_{2}, L_{3}$ are the stretches in the longitudinal, latitudinal, and thickness directions, respectively. The SBES has a stiffness of $Y=320 \mathrm{kPa}$ and an ultimate uniaxial stretch of $L_{1}=10.5$ and is a physically crosslinked elastomer (ESI Fig. S4 $\dagger$ ). Here the network is formed by a phase separation of the styrene hard blocks, with a size of approx. $50 \mathrm{~nm}$, connected by soft amorphous ethylene butylene chains. ${ }^{36}$ Consequently, the styrene domains are in the same range as the distance between LLC/QD RET pairs (of up to $20 \mathrm{~nm}$ ). ${ }^{37}$

The SEBS was dissolved in toluene at a concentration of $300 \mathrm{~g} \mathrm{~L}^{-1}$ and centrifuged for 6 hours at $6000 \mathrm{rpm}$ to remove contaminations. The PDMS was prepared as indicated by the manufacturer in a ratio of $10: 1$ directly before usage and showed a stiffness of $Y=1.6 \mathrm{MPa}$ and an ultimate stretch $L_{1}$ in the range of 1.75 up to 1.97 depending upon strain rate (ESI Fig. S5 $\dagger$ ). Both the SEBS as well as the PDMS sensors were prepared by casting $500 \mu \mathrm{L}$ of liquid elastomer onto a circular glass slide with a diameter of $25 \mathrm{~mm}$. The sample was covered and left to dry or crosslink without further treatment. After $12 \mathrm{~h}$ the sample was transferred into an oven and stored for more than two hours at $60^{\circ} \mathrm{C}$. The FRET spot was formed by drop-casting $20 \mu \mathrm{L}$ of solution containing QD655 ( $c=50 \mathrm{nM})$ and LLC $(c=500 \mathrm{nM})$ dispersed in an elastomer, onto the first elastomer layer. Consequently, the covered sensor was stored for several hours at $45{ }^{\circ} \mathrm{C}$ in an oven. The sensor spot was found to have a diameter of approx. $2 \mathrm{~mm}$. To shield the FRET spot from environmental effects $500 \mu \mathrm{L}$ of elastomer solution was drop-casted onto the stack and stored

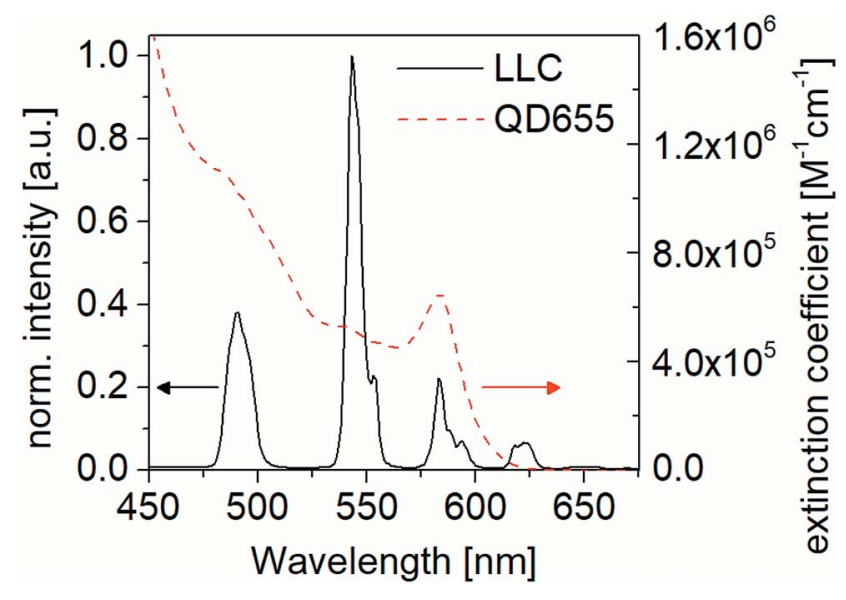

Fig. 1 Spectral overlap between LLC (donor) emission (black spectrum, scale on the left) and QD655 (acceptor) absorption (red spectrum, scale on the right). 


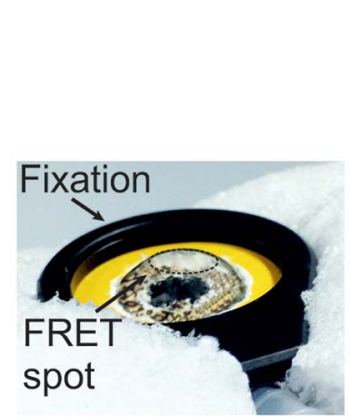

a)

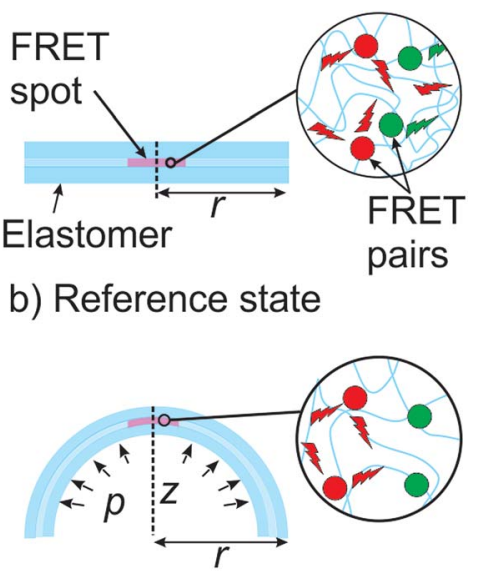

c) Pressurized state

Fig. 2 FRET pressure sensor for contactless optical readout. (a) Photograph of an inflated FRET sensor. The freestanding sensor film has a radius of approximately $r=5 \mathrm{~mm}$ and is clamped between two solid rings of cardboard. In the reference Off-state the FRET spot has a diameter of approximately $2 \mathrm{~mm}$ and is in the centre of the film. (b) Cross section of the sensor in the reference state with no applied pressure. The FRET spot is embedded in an elastomer film and characterized by high FRET efficiency. (c) Exposed to a pressure $p$ the sensor inflates out of plane and takes a hemispherical shape. The deformation yields an increase in donor-acceptor distance, which causes an increase in donor luminescence and simultaneous decrease in acceptor fluorescence because of lower FRET efficiency.

for several hours at $60^{\circ} \mathrm{C}$. The casted sensor showed a thickness of $112 \mu \mathrm{m}$ and $325 \mu \mathrm{m}$ for the SEBS and PDMS film, respectively. Finally, the sensor was removed from the glass slide and clamped between two rigid rings of a cardboard. The total diameter of the structure was $25 \mathrm{~mm}$ and defines a freestanding inner radius of $r=5 \mathrm{~mm}$ (see Fig. 2). The sample was mounted into a modified lenses holder (Newport/Thorlabs) connected to a pressure valve and gauge to perform inflation experiments. Since SEBS and PDMS are highly transparent in the VIS spectral region, we assume that absorption can be greatly neglected for all our experiments. Before transferring the sensor module into the plate reader, functionality was tested by applying a net pressure of $20 \mathrm{kPa}$ and evaluating the inflation lift $Z$ and apex stretches consequently (ESI, Fig. S1 $\dagger$ ). Inside the plate reader the sensors were altered between reference state (0 kPa - Off) and pressured state (20 kPa - On) with a holding time of $30 \mathrm{~s}$ each.

\section{Luminescence measurements}

Luminescence data were collected contactless with a plate reader (LF 500 Nanoscan, IOM, Berlin, Germany) using two photomultiplier tubes (PMT). The setup was modified to facilitate measurements of the freestanding sensor film in the reference Off- as well as the pressured On-state (ESI, Fig. S1†). The PMTs were equipped with interchangeable band pass filters (Semrock, USA) with a centre wavelength of $494 \pm 10 \mathrm{~nm}$ and $655 \pm 8 \mathrm{~nm}$ respectively to match the LLC and QD655 emission. The pressure was manually applied and monitored continuously with the connected manometer. During the steady-state measurements, no pressure decay was recognised. The FRET spot was excited from the distance of $5 \mathrm{~mm}$ by a pulsed nitrogen laser (Lasertechnik Berlin, Germany) with an emission wavelength of $337 \mathrm{~nm}$ using 128 shots at $30 \mathrm{~Hz}$ repetition rate (laser pulse duration $c a .7 \mathrm{~ns}$ ) and a pulse energy of $c a$. $30 \mu \mathrm{J}$. For luminescence measurements, a time gate of 50$450 \mu$ s was defined to avoid detection of short-lived background fluorescence. The luminescence decay times were measured at room temperature in the Off- as well as in the On-state for $10 \mathrm{~ms}$ with the temporal resolution of $10 \mu \mathrm{s}$. The average LLC luminescence decay time was calculated using the bi-exponential fitting procedure provide by FAST $^{\mathrm{TM}}$ advanced software package (Edinburgh Instruments, UK).

\section{Results \& discussion}

LLC/QD pairs are currently the best match for the elastic pressure sensor due to their unique photo physical properties. LLC is expected to show long luminescence lifetimes ( $\mu \mathrm{s}-\mathrm{ms}$ range) for nearly background-free luminescence measurements by time-gated detection while QD655 is known to provide sufficient photo stability, brightness and detection limits down to $\mathrm{fM} \cdot{ }^{38-41}$

\section{Sensor validation - time-gated steady-state luminescence}

It should be noted that preliminary inflation experiments without encapsulation of the sensor spot, a non-neglectable decay of efficiency (data not shown) was recognised. To ensure that the encapsulation is stable, all subsequent experiments were performed in air as well as nitrogen atmosphere. Our measurements indicate that the encapsulation shields the sensor sufficiently, since no decay of emission intensity had been noticed during all presented experiments and continuous sensor testing for more than 8 hours. The time series of donor emission (LM) and acceptor emission (FL) intensities for an alternating net pressure $p$ are shown in Fig. 3(a) and (b), respectively. Here, pressure induced geometric changes of the SEBS sensor results in an increase of LM signal intensity of and a simultaneous decrease of the FRET-induced FL intensity. The standard deviations were calculated from at least three measurements on independent samples. The donor intensity $I_{\mathrm{LM}}$ emission of the SEBS sensor is plotted in Fig. 3(a) and shows a ratio of $I_{\mathrm{LM}, \mathrm{On}, 1} / I_{\mathrm{LM}, \mathrm{Off}, 1}=1.13$ for the first Off/On cycle but also a steady decline to approx. $I_{\mathrm{LM}, \mathrm{On}, 5} / I_{\mathrm{LM}, \text { Off }, 5}=1.02$ for the 5 th cycle. Obviously, the intensity decline in short term (30 s) is significantly lower compared to the signal decline over the full duration of the experiment (600 s). These observations are likely to be explained by several parallel running processes, such as photo bleaching, donor decomposition and creep mechanics of the inflated SEBS sensor. The FRET-sensitized acceptor emissions (Fig. 3(b)) are characterized by a high reversibility and a neglectable intensity decrease either in isobaric intervals of $30 \mathrm{~s}$ as well as for all On- or all Off-states. Hence the net pressure causes an FL intensity ratio of approx. $I_{\mathrm{FL}, \mathrm{On}} / I_{\mathrm{FL}, \mathrm{Off}}=0.67$ which recovers as the pressure is released. We assume that the reversibility is mainly due to the elasticity and low hysteresis of 

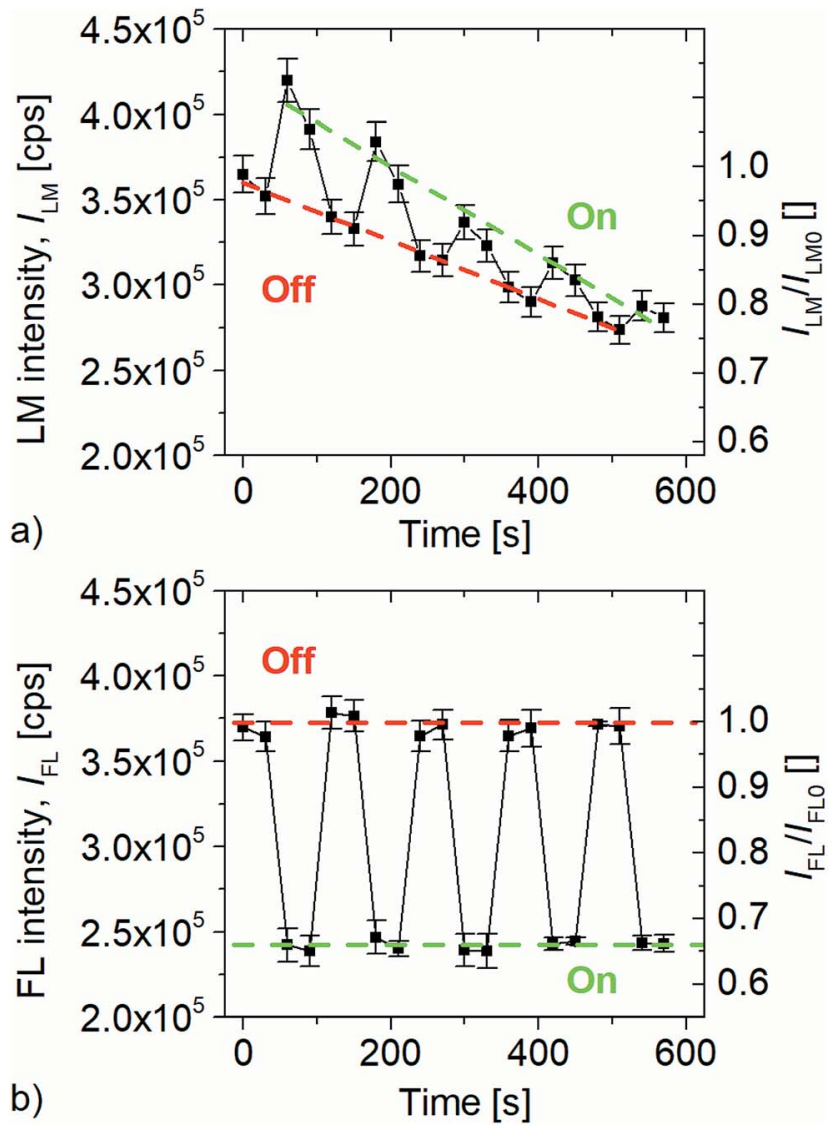

Fig. 3 Time series for alternating pressure states (Off/On) imposed on a FRET sensor with LLC/QD-pairs dispersed in a styrene-ethylene/ buthylene-styrene (SEBS)-matrix, (a) luminescence (LM) intensity of terbium complex FRET donors. (b) Fluorescence (FL) intensity of FRETsensitized emission of QD655 FRET acceptors.

the SEBS matrix (Fig. S5 $\dagger$ ). As is seen, the sensor allows a contactless readout and is capable to capture the pressure signal including the stable phase. PDMS sensors did not show any FRET emission (Fig. S2 $\dagger$ ) most likely due to unwanted interaction during the curing step.

The FL intensity ratios, affected by impreciseness in preparing identical experimental conditions over the full duration of the measurement, were compared to the pressure induced geometric changes of both sensors. We estimate conservatively that the apex region of the SEBS sensor is stretched biaxially to $1.45 \times 1.45$ causing a thickness stretch of $L_{3 \text {,SEBS }}=0.47$. Due to the stiffness of PDMS and higher sensor thickness the net pressure induce a biaxially stretch of $1.10 \times$ 1.10 and consequently a thickness stretch of $L_{3 \text {,PDMS }}=0.82$. The photophysical characterisation of the PDMS sensor indicates that the (Fig. S2 $\dagger$ ) FL intensity changes scales directly with the thickness stretch $L_{3, \text { PDMs }}$ during inflation. This assumption holds as the area of the pump spot remains unchanged (ESI, Fig. S2 $\uparrow$ and 3). Nevertheless, for the SEBS sensor the FL intensity ratio is $I_{\mathrm{FL}, \mathrm{On}, 1} / I_{\mathrm{FL}, \mathrm{Off}, 1} \sim 0.65$ which is significantly higher than the corresponding thickness stretch $L_{3 \text {,SEBS }}$. The mismatch is to be explained with contribution of FRET from LLC to QD which leads to a higher FL intensity of the QD. This is further supported by the time-resolved measurements. The standard deviations of all short-term FL intensity ratios in the pressured as well as unpressured states are $13 \times 10^{-3}$ and 8.12 $\times 10^{-3}$ respectively. Both values are very similar and indicate repeatability but also durability of the samples when operated as pressure sensors with large geometric deformations. Evidently, the decay of donor emissions hardly affects the acceptor emission (Fig. 3(b)) which is expected, due to a ratio of $10: 1$ for LLC to QD. The high ratio ensures that the average number of LLC/QD-pairs and thus the FRET-sensitized acceptor emission stays virtually constant and a donor molecule is being always in vicinity of a QD.

The pressure sensitivity is evaluated using the relative unit cps $\mathrm{kPa}^{-1}$. The cps unit refers to the number of photons per second counted by the photomultiplier. Considering that the net pressure is $20 \mathrm{kPa}$ and the associated change in FL intensity is approx. $127.000 \mathrm{cps}$, the calculated sensor response is ca. $6350 \mathrm{cps} \mathrm{kPa}^{-1}$. However, with the chosen measurement setup the accuracy of pressure measurement is $0.6 \mathrm{kPa}$ which corresponds to $3810 \mathrm{cps}$.

The shelf life of the elastic FRET sensors was not investigated during this project. Generally, the shelf life is hard to predict since the shelf life of individual sensor materials (polymers, QDs and LLC) strongly depends on the storing conditions and can be in the range of months up to years, but has not been explicitly tested during this work. Unfortunately, it was also not possible to evaluate the complex interplay between chemicals, photo-induced mechanisms and large geometric deformations affecting the FRET lifetime during this work.

\section{Sensor validation - time-resolved luminescence}

Changes in signal intensity due to FRET are accompanied by changes in the luminescence decay time of the donor and acceptor signal. In general, a high FRET efficiency is associated with a short luminescence decay time of the donor signal which allows to detect changes of FRET efficiency using time-resolved luminescence. The LLC/QD (FRET) pair provides a luminescence decay time in the range of milliseconds for the LLC, whereas the decay time of the QD is in the nanosecond range. In case of FRET taking place from such a LLC donor to a QD acceptor it is likely to assume that the FRET efficiency acceptor luminescence decay time is equal to the donor luminescence decay time. ${ }^{42}$ FRET-sensitized emission spectra of QD (acceptors in Fig. 4(a) and (b)) shows a decay in the range of $\mu$ s up to ms, which agrees well with our assumptions. Therefore, the higher the FRET efficiency the longer will be the luminescence decay time of acceptor quantum dots.

The donor and acceptor luminescence decays in Fig. 4 evidently show that decay time of donor luminescence increases, whereas the decay time of the acceptor channel decreases as the net pressure is applied. For the donor channel the average decay time is equal to $270 \mu \mathrm{s}$, which increases to 420 $\mu \mathrm{s}$ in the pressured state. Simultaneously, the average decay time in the acceptor channel decreases from $170 \mu \mathrm{s}$ to $155 \mu \mathrm{s}$. The above results successfully demonstrate that the timeresolved measurements can be used to discriminate the 


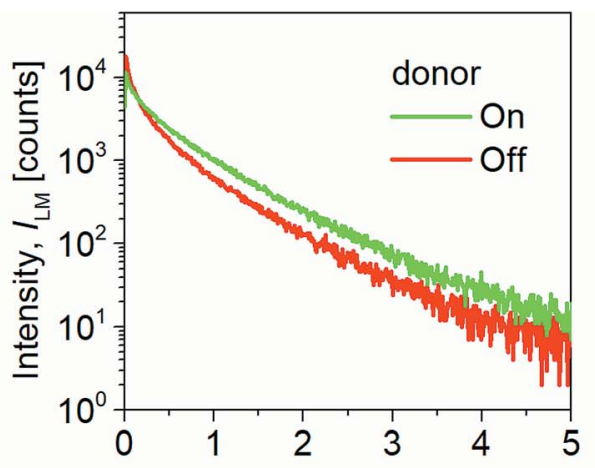

a)

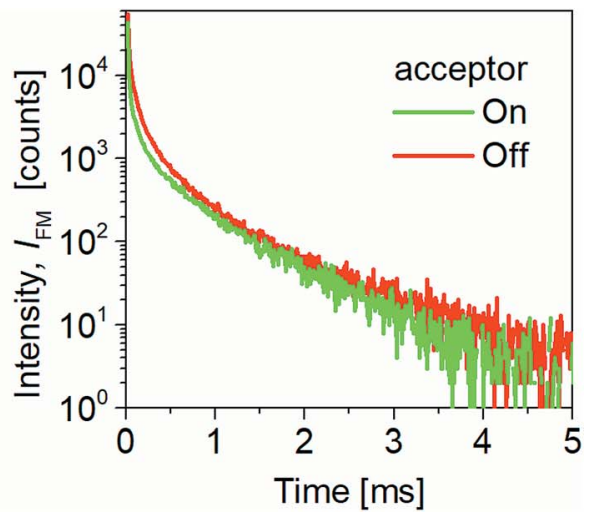

b)

Fig. 4 Luminescence decay measurements of LLC (donor) and QD655 (acceptor) molecules incorporated in SEBS for the reference (OFF-state) and the pressured state (ON-state). In the OFF-state the FRET-donors and acceptors are in closer proximity while the ON-state causes larger distances and consequently a reduced FRET-efficiency. (a) Time traces of donor intensity (b) time traces of acceptor intensity.

pressure levels with the FRET-based sensor. The calculated lifetime and intensity of acceptors are higher in the Offcompared to the On-state. All luminescence decays are of multiexponential kind since donor and acceptor molecules are randomly distributed inside the elastomer matrix, resulting in a distance distribution of all LLC/QD FRET pairs with consequence on the FRET efficiency.

\section{Conclusions}

We conclude that FRET induced luminescence of donor/ acceptor pairs incorporated in an elastic matrix can be used to monitor net pressure changes. The contactless sensor readout has been demonstrated using steady-state as well as timeresolved luminescence spectroscopy. The elastic sensors were prepared by dispersing donor/acceptor pairs (LLC/QD) in a thermoplastic triblock copolymer or PDMS. Tested materials and sensors reveal that the acceptor emission scale with the applied net pressure and consequently with the corresponding geometric changes. The long-term decline $(600 \mathrm{~s})$ in donor intensities was found to be higher than in the short-term (30 s), whereas the acceptor emission intensity remained constant over time. Consequently, the drift in the emission due to changes in acceptor luminescence could cause problems for the exact determination of pressure or geometric changes using this material. However, for biomedical applications but also competing electronic applications such as skin-like electronics, only the short-term changes are important. Proposed FRET active composite and the contactless time as well as space resolved monitoring allows for a dramatic miniaturisation and sensor complexity, otherwise harder to achieve with regular techniques and materials proposed for flexible sensors and electronics. Nevertheless, neither the material composition nor the sensor design and production have been optimized so far. Therefore, it is likely that sensor improvement in terms of accuracy, durability and lifetime can be achieved directly. To exploit the full potential of FRET, further work is being pursued to extend the selection of suitable FRET pairs and host materials to match requirements of specific applications. These attempts might be extended to gain additional sensor functionality by functionalisation of the FRET pairs. Among our very next steps to enhance the signal accuracy and stability, sealing materials and sealing techniques will be improved to reduce the donor emission decay.

\section{Conflicts of interest}

There are no conflicts to declare.

\section{Acknowledgements}

AG, FM, MB thank the Fraunhofer Gesellschaft for the "Nanopolyphotonic" seed funding. MK acknowledges the German Federal Ministry of Education and Research (BMBF) for support via Grant No. 03X5511 "KompAkt" (WING-NanoFutur). MP and KG thank the National Science Centre Grant No. 2015/17/B/ST5/ 01038.

\section{References}

1 M. Kaltenbrunner, T. Sekitani, J. Reeder, T. Yokota, K. Kuribara, T. Tokuhara, M. Drack, R. Schwödiauer, I. Graz, S. Bauer-Gogonea, S. Bauer and T. Someya, Nature, 2013, 499, 458.

2 D. Kim, R. Ghaffari, N. Lu and J. A. Rogers, Annu. Rev. Biomed. Eng., 2012, 14, 113.

3 J. A. Rogers, T. Someya and Y. Huang, Science, 2010, 327, 1603.

4 T. Someya, Stretchable Electronics, Wiley-VCH, Weinheim, Germany, 2013.

5 C. Weder, J. Mater. Chem., 2011, 21, 8235.

6 H. Stoyanov, M. Kollosche, S. Risse, R. Wache and G. Kofod, Adv. Mater., 2013, 25, 578.

7 I. Kang, M. J. Schulz, J. H. Kim, V. Shanov and D. Shi, Smart Mater. Struct., 2006, 15, 737.

8 C. Jo, D. Pugal, I. K. Oh, K. J. Kim and K. Asaka, Prog. Polym. Sci., 2013, 38, 1037. 
9 M. L. Hammock, A. Chortos, B. C. K. Tee, J. B. H. Tok and Z. Bao, Adv. Mater., 2013, 25, 5997.

10 D.-H. Kim, N. Lu, R. Ma, Y.-S. Kim, R.-H. Kim, S. Wang, J. Wu, S. M. Won, H. Tao, A. Islam, K. J. Yu, T. Kim, R. Chowdhury, M. Ying, L. Xu, M. Li, H.-J. Chung, H. Keum, M. McCormick, P. Liu, Y.-W. Zhang, F. G. Omenetto, Y. Huang, T. Coleman and J. A. Rogers, Science, 2011, 333, 838.

11 J. Brownjohn, Philos. Trans. R. Soc., A, 2007, 365, 589-622.

12 S. Laflamme, M. Kollosche, J. J. Connor and G. Kofod, Struct Contr. Health Monit., 2012, 19, 70.

13 J. A. Rogers, T. Someya and Y. Huang, Science, 2010, 327, 1603.

14 D. H. Kim, Z. Liu, Y. S. Kim, J. Wu, J. Song, H. S. Kim, Y. Huang, K.-c. Hwang, Y. Zhang and J. A. Rogers, Small, 2009, 5, 2841.

15 J. T. Muth, D. M. Vogt, R. L. Truby, Y. Mengüç, D. B. Kolesky, R. J. Wood and J. A. Lewis, Adv. Mater., 2014, 26, 6307.

16 M. Drack, I. Graz, T. Sekitani, T. Someya, M. Kaltenbrunner and S. Bauer, Adv. Mater., 2015, 27, 34.

17 H. Stoyanov, M. Kollosche, D. N. McCarthy and G. Kofod, J. Mater. Chem., 2010, 20, 7558.

18 M. Kollosche, H. Stoyanov, S. Laflamme and G. Kofod, J. Mater. Chem., 2011, 21, 8292.

19 A. Ryabchun, M. Wegener and O. Sakhno, RSC Adv., 2016, 6, 51791.

20 A. Ryabchun, M. Kollosche, M. Wegener and O. Sakhno, Adv. Mater., 2016, 28, 10217.

21 M. Swierczewska, G. Liu, S. Lee and X. Chen, Chem. Soc. Rev., 2012, 41, 2641.

22 T. Förster, Naturwissenschaften, 1946, 33, 166.

23 T. Förster, Ann. Phys., 1948, 437, 55.

24 T. Förster, Discuss. Faraday Soc., 1959, 27, 7.

25 K. E. Sapsford, L. Berti and I. L. Medintz, Angew. Chem., 2006, 118, 4676.

26 P. R. Selvin, Nat. Struct. Biol., 2000, 7, 730.
27 H. Bazin, M. Preaudat, E. Trinquet and G. Mathis, Spectrochim. Acta, Part A, 2001, 57, 2197.

28 L. Stryer, Annu. Rev. Biochem., 1978, 47, 819.

29 L. Stryer and R. P. Haugland, Proc. Natl. Acad. Sci. U. S. A., 1967, 58, 719.

30 Y. Niino, K. Hotta and K. Oka, PLoS One, 2009, 4, e6036.

31 S. M. Cohen, M. Meyer and K. N. Raymond, J. Am. Chem. Soc., 1998, 120, 6277.

32 L.-N. Sun, J. Yu, H. Peng, J. Z. Zhang, L.-Y. Shi and O. S. Wolfbeis, J. Phys. Chem. C, 2010, 114, 12642.

33 J. R. Lakowicz in Principles of Fluorescence Spectroscopy, Springer Science + Business Media, New York, 3rd edn, 2006, p. 443, 13 Energy Transfer.

34 B. W. van der Meer, G. Coker and S. Y. S. Chen, Resonance Energy Transfer: Theory and Data, VCH Publishers Inc., New York, 1994.

35 J. Laurer, R. Bukovnik and R. Spontak, Macromolecules, 1996, 29, 5760 .

36 Y. A. Tang, J. Hu, A. H. Elmenoufy and X. L. Yang, ACS Appl. Mater. Interfaces, 2015, 7, 12261.

37 L. Olejko, P. J. Cywinski and I. Bald, Angew. Chem., Int. Ed., 2015, 54, 673.

38 F. Morgner, D. Geissler, S. Stufler, N. G. Butlin, H.-G. Löhmannsröben and N. Hildebrandt, Angew. Chem., Int. Ed., 2010, 49, 7570.

39 F. Morgner, S. Stufler, D. Geissler, I. L. Medintz, W. R. Algar, K. Susumu, M. H. Stewart, J. B. Blanco-Canosa, P. E. Dawson and N. Hildebrandt, Sensors, 2011, 11, 9667.

40 P. J. Cywinski, T. Hammann, D. Huhn, W. J. Parak, N. Hildebrandt and H.-G. Löhmannsröben, J. Biomed. Opt., 2014, 19, 101506.

41 P. J. Cywinski, K. N. Nono, L. J. Charbonniere, T. Hammann and H.-G. Löhmannsröben, Phys. Chem. Chem. Phys., 2014, 16, 6060 .

42 B. Valeur, Molecular Fluorescence: Principles and Applications, Wiley-VCH, New York, 2002. 